Proceedings of SALT 23: 358-375, 2013

\title{
Diagnosing truth, interactive sincerity, and depictive sincerity *
}

\author{
Elizabeth Coppock \\ University of Gothenburg
}

\author{
Thomas Brochhagen \\ University of Düsseldorf
}

\begin{abstract}
This paper presents two experimental findings pertaining to the semantics and pragmatics of superlative modifiers (at least, at most). First, in a scenario with $N$ objects of a given type, speakers consistently judge it true that there are 'at least $N$ ' and 'at most $N$ ' objects of that type. This supports the debated position that the ignorance conveyed by superlative modifiers is an implicature, not an entailment, and contrasts with results obtained using an inference-judgment paradigm, suggesting that truth-value judgment tasks are impervious to certain pragmatic infelicities that inference-judgment tasks are sensitive to. The second finding is not predicted by any previous theory: In a scenario with $N$ objects, it is not consistently judged true that there are 'at most $N+1$ ' objects, even though it is consistently judged true that there are 'at least $N-1$ ' objects. To explain this, we propose a novel pragmatic principle requiring that the scenario depicted by a sentence must be considered possible by the speaker (the Maxim of Depictive Sincerity). Put together, the two findings show that truth-value judgment tasks are impervious to some aspects of pragmatics, but not all.
\end{abstract}

Keywords: modified numerals; ignorance implicatures; inquisitive semantics; highlighting; experimental methodology

\section{The ignorance implication}

A well-established fact about superlative modifiers is that they convey ignorance on the part of the speaker (see, for example, Geurts \& Nouwen 2007, Büring 2008 and Nouwen 2010). This is not the case for comparatives, as illustrated by the following contrast:

(1) a. Exactly eight students applied for the position, so that's more than four.

b. Exactly eight students applied for the position, \#so that's at least five.

In (1a), the speaker establishes that he or she knows how many students applied for the position, and subsequently describes the quantity as more than four. This can

* We would especially like to thank Matthijs Westera and Laura Staum Casasanto for feedback on this work, as well as the audiences at SALT 2013 and Gothenburg Workshop on Questions and Inquisitive Semantics. Research at the University of Gothenburg was supported by Swedish Research Council grant Semantic Analysis of Interaction and Coordination in Dialogue. 
be done without raising any eyebrows. But example (1b) is infelicitous because the ignorance implied by at least five clashes with the speaker's knowledge as to the number of applicants.

The existence of the ignorance implication has further been corroborated by the experimental findings of Geurts, Katsos, Cummins, Moons \& Noordman (2010). In one of their experiments, participants were asked to judge the validity of inferences from bare numerals (as in (2a)) to modified numerals (as in (2b)).
a. Berta had $N$ beers.
[Premise]
b. Berta had $\left\{\begin{array}{l}\text { at least } N \\ \text { more than } N-1 \\ \text { at most } N \\ \text { fewer than } N+1\end{array}\right\}$ beers.
[Conclusion]

Their results showed a significant difference between comparatives and superlatives. While all participants accepted the inference for more than and fewer than, only around half accepted it for at least and at most. This contrast can be explained under the assumption that at least and at most express ignorance, which conflicts with the certainty expressed by the bare numeral in the premise.

Geurts et al. (2010) interpret the findings of their inference judgment task as evidence for the view advocated by Geurts \& Nouwen (2007), according to which the ignorance implication is an entailment. Geurts \& Nouwen (2007) propose that superlative modifiers have the following modal meaning, where the ignorance implication is expressed by the epistemic possibility operator (signified here by ' $\diamond$ '; ' $\square$ ' signifies epistemic necessity).

(3) a. Berta had at least three beers.

b. $\square[$ Berta had three beers $] \wedge \diamond$ [Berta had more than three beers $]$

a. Berta had at most three beers.

b. $\diamond[$ Berta had three beers $] \wedge \square$ [Berta had no more than three beers $]$

We refer to this as the 'ignorance-as-entailment view'.

A more pervasive view (Büring 2008; Cohen \& Krifka 2011; Coppock \& Brochhagen 2013, i.a.) is that the ignorance inference is a conversational implicature. Geurts et al.'s (2010) results are compatible with this view as well. As Kaplan (1999) points out, validity intuitions are determined by whether the information conveyed by the conclusion is contained in the information conveyed by the premise, and truth-conditions are not the only kind of information that may play a role in such a setting. In our case, the ignorance implicature conveyed by the conclusion is not contained in the premise. The ignorance-as-implicature view moreover sheds light on why $50 \%$ of the participants did judge the inference to hold 
for at least. On the ignorance-as-implicature view, the inference is logically valid, even though the conclusion is pragmatically infelicitous when the premise is true.

Hence, Geurts et al.'s results are compatible with both views, and further data is needed in order to tease them apart. We used a slightly different methodological paradigm for this purpose. Rather than asking participants to judge whether $\mathrm{A}$ implies B, we depicted a scenario where A is true, and asked them whether B is true or false in that scenario. This methodology turned out, as we expected, to diagnose entailment more precisely than the validity-judgment task: in a scenario with $N$ objects of a given type, we found that speakers consistently judge it true that there are 'at least $N$ ' and 'at most $N$ ' objects of that type (and we found no contrast with comparative modifiers). We will report on those results in detail in $\S 3$, after briefly describing Coppock \& Brochhagen's (2013) particular ignorance-as-implicature analysis, which uses inquisitive semantics.

However, this is not the end of the story. We found an additional result that is not predicted by any previous theory: In a scenario with $N$ objects, it is not consistently judged true that there are 'at most $N+1$ ' objects, even though it $i s$ consistently judged true that there are 'at least $N-1$ ' objects. To explain this, we propose a novel pragmatic principle requiring that the scenario depicted by a sentence must be considered possible by the speaker (the Maxim of Depictive Sincerity). We adopt the notion of 'highlighting' from inquisitive semantics in order to formalize this principle.

Methodologically, these results have two broader consequences, taken together: In contrast to validity-judgment tasks, truth-value judgment tasks are impervious to some pragmatic violations, but there are still some pragmatic infelicities that truth-value judgment tasks are sensitive to.

\section{Ignorance as implicature}

As mentioned above, there are a number of different analyses on which the ignorance implication is treated as an implicature. In this section, we summarize Coppock \& Brochhagen's (2013) particular instantiation of this view, which uses inquisitive semantics. This is the analysis we will propose to build upon later in the paper.

Coppock \& Brochhagen's analysis takes inspiration from Büring's (2008) idea that superlative modifiers are very much like disjunctions. In particular, just like superlative modifiers, disjunctions can evoke ignorance implicatures, as has been much discussed in recent literature. For example, disjunctions behave similarly to superlative modifiers in a context like the one in (1). Consider (5).

Exactly eight students applied for the position, \#so that's eight or more.

Büring (2008) proposes to treat at least $\alpha$ as a disjunction over alternatives ranked as 


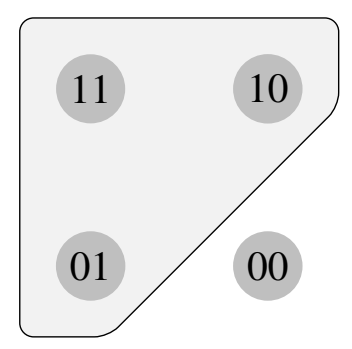

(a) Classical

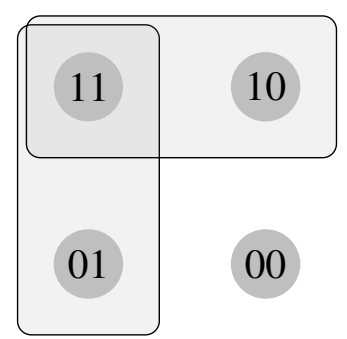

(b) Inquisitive

Figure 1 Classical and inquisitive disjunction.

high or higher than $\alpha$ on a pragmatically-given scale; Cummins \& Katsos 2010 and Biezma 2013 adopt this analysis as well. Rather than treating superlative modifiers as disjunctions (which becomes problematic when one attempts to specify the level at which this classification holds), Coppock \& Brochhagen propose that superlative modifiers and disjunctions have an important property in common, namely that they are both typically inquisitive, in the sense of inquisitive semantics. (The reader is referred to that paper for justification of this approach.)

In contrast to classical frameworks, where a declarative sentence denotes a set of possible worlds and an interrogative sentence denotes a set of sets of possible worlds, both declaratives and interrogatives denote sets of sets of possible worlds in inquisitive semantics. If, following inquisitive semantics jargon, we use the term possibility for a set of possible worlds, we can say that both declarative and interrogative sentences denote sets of possibilities in inquisitive semantics. The sets of possibilities that sentences may denote are called propositions.

To illustrate, let us consider how disjunctions are treated in both cases. In inquisitive semantics, a disjunction denotes a set of possiblities, one per disjunct, each containing all the worlds under which the respective disjunct is true. Under a classical analysis, in contrast, disjunctions are treated as an undifferentiated set of possible worlds. The contrast between classical and inquisitive disjunctions is shown in Figure 1, where each dot represents a possible world, and the numbers on the dots represent what is true at the world. For example, let $w_{10}$ be the world where Ann snores and Bill does not, $w_{01}$ the world where Bill snores and Ann does not, $w_{11}$ the world where both snore and $w_{00}$ the world where neither of them snores. Then the disjunction being represented is Ann or Bill snores.

Coppock \& Brochhagen (2013) make use of an extension of the original inquisitive semantics framework called unrestricted inquisitive semantics (Ciardelli, Groenendijk \& Roelofsen 2009, 2012). In the original framework, propositions 
contain all subsets of every possibility they contain, but in unrestricted inquisitive semantics, this property is not required to hold, so nested possibilities can play an important role. In this setting, a proposition is said to be inquisitive if it contains more than one maximal possibility, where a maximal possibility is a possibility that is not fully contained by any other possibility. A proposition is attentive if it contains a non-maximal possibility (the idea being that one draws attention to each of the possibilities in the denotation).

(6) Inquisitivity and attentiveness (Ciardelli et al. 2009)

a. $\varphi$ is inquisitive iff $\llbracket \varphi \rrbracket$ contains at least two maximal possibilities;

b. $\varphi$ is attentive iff $\llbracket \varphi \rrbracket$ contains a non-maximal possibility.

For the discussion of superlative modifiers, it is useful to have a term covering both:

\section{Interactivity (Coppock \& Brochhagen 2013)}

$\varphi$ is interactive iff $\llbracket \varphi \rrbracket$ contains more than one possibility.

A proposition is interactive iff it is inquisitive or attentive.

Coppock \& Brochhagen's (2013) claim is that sentences with superlative modifiers are, in general, interactive. Specifically, Coppock \& Brochhagen propose that at least $\alpha$ denotes the set of possibilities that are as strong or stronger than $\llbracket \alpha \rrbracket$, and that at most $\alpha$ denotes the set of possibilities weaker than or as strong as $\llbracket \alpha \rrbracket$, excluding higher ranked possibilities. The possibilities are answers to the current question under discussion (QUD) ranked by pragmatic strength $(\leq)$. For the present purpose, Coppock \& Brochhagen's (2013) proposed meanings of superlatives can be simplified as follows ( $s$ stands for 'state' and represents the current discourse context):

$$
\begin{aligned}
& \llbracket \text { at least } \alpha \rrbracket^{s}=\left\{p \mid p \leq_{s} p^{\prime} \text { for some } p^{\prime} \in \llbracket \alpha \rrbracket^{s}\right\} \\
& \llbracket \text { at most } \alpha \rrbracket^{s}=\left\{p^{\prime} \cap \operatorname{MAX}_{s}(p) \mid p \in \llbracket \alpha \rrbracket^{s} \wedge p^{\prime} \leq_{s} p\right\} \\
& \text { where } \operatorname{MAX}_{s}(p)=\lambda w . \forall p^{\prime} \in \operatorname{QUD}_{s}: p^{\prime}(w) \rightarrow p \geq_{s} p^{\prime}
\end{aligned}
$$

To take a simple example, consider At least Ann snores vs. Ann snores in the context of the QUD 'Who snores?', depicted in Figure 2. The information provided by the two sentences is identical, but the former is interactive. At least Ann snores denotes the set of possibilities as strong as or stronger than Ann snores. This contains the possibility of Ann snoring, $\left\{w_{10}, w_{11}\right\}$, and the possibility that both Ann and Bill snore, $\left\{w_{11}\right\}$. The denotation of plain, unadorned Ann snores contains just one possibility, that of Ann snoring. The relationship between At least Ann snores and Ann snores is analogous to the relationship between inquisitive and classical disjunction: same underlying set of worlds, but more structure in the former.

As modified numerals will figure prominently in the experiments to be reported on later, let us illustrate how they work. 
Diagnosing truth, interactive sincerity, and depictive sincerity

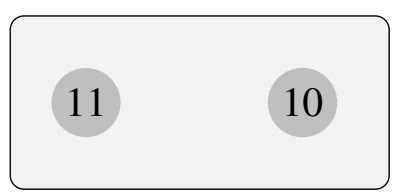

01

\section{0}

(a) Ann snores

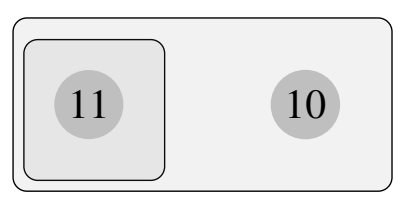

01

(b) At least Ann snores

Figure 2 Interactive and non-interactive propositions.

QUD: How many beers did Ann drink?

a. Ann drank three beers.

b. Ann drank three or four beers.

c. Ann drank at least three beers.

d. Ann drank at most three beers.

Let us assume that numerals like three have a 'one-sided' semantics, so three is semantically equivalent to three or more. Further, assume that there is exactly one world $w_{n}$ for every number of beers $n$ that Ann may have consumed. Let $p_{n}=\left\{w_{n}, w_{n+1}, \ldots\right\}$. For the strength ranking we assume $p_{m}>_{s} p_{n}$ iff $m>n$. Then the sentences in (10) will receive the following interpretations:

a. $\llbracket$ Ann drank 3 beers $\rrbracket^{s}=\left\{p_{3}\right\}=\left\{\left\{w_{3}, w_{4}, \ldots\right\}\right\}$

b. $\llbracket$ Ann drank 3 or 4 beers $\rrbracket^{s}=\left\{p_{3}, p_{4}\right\}=\left\{\left\{w_{3}, w_{4}, \ldots\right\},\left\{w_{4}, w_{5}, \ldots\right\}\right\}$

c. $\llbracket$ Ann drank at least 3 beers $\rrbracket^{s}=\left\{p \mid p \geq_{s} p_{3}\right\}$ $=\left\{\left\{w_{3}, w_{4}, \ldots\right\},\left\{w_{4}, w_{5}, \ldots\right\},\left\{w_{5}, w_{6}, \ldots\right\}, \ldots\right\}$

d. $\llbracket$ Ann drank at most 3 beers $\rrbracket^{s}=\left\{p \cap\left\{w_{0}, \ldots, w_{3}\right\} \mid p \leq_{s} p_{3}\right\}$ $=\left\{\left\{w_{0}, \ldots, w_{3}\right\},\left\{w_{1}, w_{2}, w_{3}\right\},\left\{w_{2}, w_{3}\right\},\left\{w_{3}\right\}\right\}$

Just as disjunctions denote one possibility per disjunct, both at least and at most denote one possibility for each alternative under consideration. Thus, the sentences with superlative modifiers are interactive according to the definition in (7).

To account for the ignorance implicature, Coppock \& Brochhagen (2013) generalize Groenendijk \& Roelofsen's (2009) Maxim of Inquisitive Sincerity as follows:

(12) Maxim of Interactive Sincerity (Coppock \& Brochhagen 2013)

If $\varphi$ is interactive, then $\varphi$ is interactive in the speaker's information set. 
The speaker's information set contains just those possible worlds that are epistemically accessible to the speaker, and a proposition is interactive there if it is interactive once restricted to those worlds. This maxim requires that, if a speaker draws attention to multiple possibilities (as is the case for sentences involving superlatives), she must not know which of the possibilities under consideration holds: 'Don't raise an issue if you already know how to settle it.' Thus, in general, if a speaker knows an alternative expressed by $\alpha$ to hold, it is infelicitous to utter at least $\alpha$.

Comparatives are not interactive according to Coppock \& Brochhagen (2013) and are therefore not subject to the Maxim of Interactive Sincerity. Coppock \& Brochhagen (2013) assume that more than $\alpha$ denotes a singleton set whose sole element is the union of all alternatives ranked higher than $\alpha$. So Ann drank more than two beers would have the following denotation.

(13) $\llbracket$ Ann drank more than two beers $\rrbracket^{s}=\left\{\bigcup\left\{p \mid p>_{s} p_{2}\right\}\right\}=\left\{\left\{w_{3}, w_{4}, \ldots\right\}\right\}$

A speaker who knew exactly how many beers Ann drank would not be in violation of the Maxim of Interactive Sincerity for using this sentence. Hence the contrast between the sentences in (1).

\section{Experiments}

The two hypotheses about the nature of the ignorance implication make different predictions. Under the ignorance-as-entailment view, exactly $N$ does not entail at least $N$, and if it is known to the speaker that there are exactly three apples, then There are at least three apples should be judged as false. On the ignorance-as-implicature view, exactly $N$ entails at least $N$. This latter view predicts There are at least three apples to be judged true if there are obviously exactly three apples, as long as the judgment is really made on the basis of truth and not pragmatic (in)felicity.

The inference judgment task of Geurts et al. (2010) was designed to get at this contrast. Asking participants to judge if exactly $N$ implies at least $N$ seems indeed like a straightforward way to determine whether exactly $N$ entails at least $N$. But, as we argued above, this methodology may fail to detect entailments when the conclusion gives rise to an implicature that is not conveyed by the premise. Therefore, we conducted experiments asking for truth-value judgments instead. In these experiments, we depicted a scenario with exactly $N$ objects, and asked participants to judge sentences as true or false with respect to the depicted scenario.

\subsection{Experiment 1}

The first experiment tested whether, for example, There are at least three apples and There are at most three apples are judged to be true in a situation with three apples. 


\subsubsection{Methods}

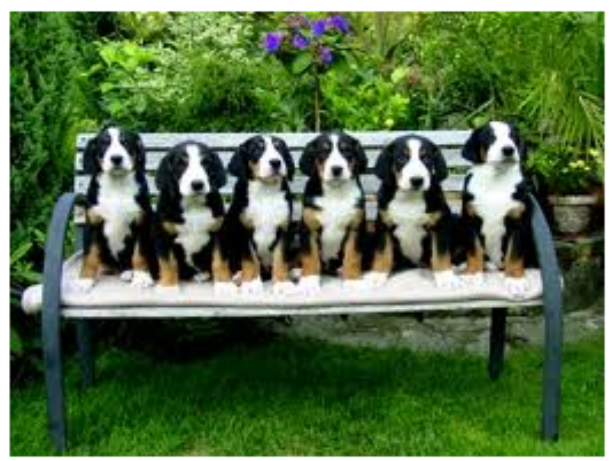

There are at least 6 dogs in the picture.

True

False

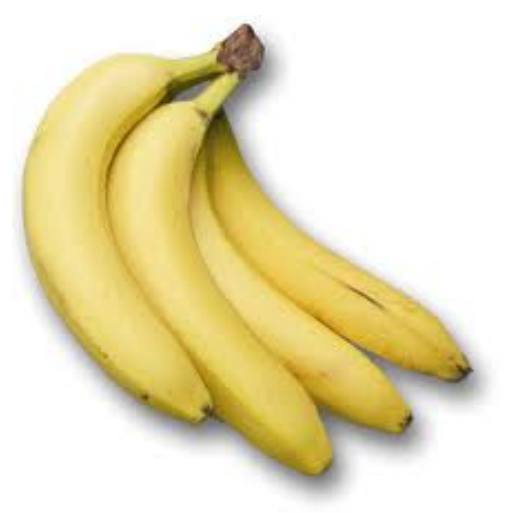

There are at most 3 bananas in the picture.

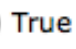

False

Figure 3 Experimental stimuli from Experiment 1.

Forty participants, recruited through Amazon's Mechanical Turk, were presented with a series of sentences paired with pictures, and asked to judge for each sentence whether it was true or false with respect to the picture. Each picture contained three, four, five, or six objects of a given type (three bananas, four boats, etc.). There were 32 pictures in total, with eight of each quantity between three and six. Examples are shown in Figure 3.

These pictures were rotated via a latin square design through four critical and four control conditions, so that no participant saw a given picture more than once, and every picture was seen in every condition. In the control conditions, the sentences were predicted to be judged false (e.g. There are more than three bananas in the picture when the picture only showed three bananas). For a picture with $N$ objects, the critical conditions were at least $N$ and at most $N$, more than $N-1$ and less than $N+1$. The eight conditions are shown in Table 1 .

Each condition was judged four times per participant, with four different pictures, one for each quantity of objects. In total, each participant saw 32 experimental stimuli and 32 fillers. The fillers also involved counting objects (e.g. There are exactly four forks in the picture). 


\begin{tabular}{ll}
\hline \multicolumn{1}{c}{ Critical } & \multicolumn{1}{c}{ Control } \\
\hline There are at most $N X \mathrm{~s}$ in the picture. & There are at most $N-1 X \mathrm{~s}$ in the picture. \\
There are fewer than $N+1 X \mathrm{~s}$ in the picture. & There are fewer than $N X \mathrm{~s}$ in the picture. \\
There are at least $N X \mathrm{~s}$ in the picture. & There are at least $N+1 X \mathrm{~s}$ in the picture. \\
There are more than $N-1 X \mathrm{~s}$ in the picture. & There are more than $N X \mathrm{~s}$ in the picture.
\end{tabular}

$N=$ amount of objects depicted, $X=$ type of object depicted.

Table 1 Conditions used in Experiment 1.

\subsubsection{Results and discussion}

As expected, the control sentences were reliably judged to be false. More importantly, no significant difference between the critical conditions was found, as every sentence type was almost unanimously judged to be true. The percentage of "true" judgments for each condition is shown in Table 2 and graphed in Figure 4. As the reader may expect given the extremely high percentages of "true" judgments in every condition, there is no significant difference between the conditions (based on a mixed-effects binomial logit model with dummy-coded variables for each of the four conditions and random effects for subject and item; $p>0.5$ for each pairwise contrast, according to the $t$-statistic). Since there were so many observations per condition, this study had sufficient power to detect a difference as little as three percentage points if it were there; cf. the height of the error bars in Figure 4.

These results support the hypothesis that exactly $N$, represented by the objects in the pictures, in fact entails both at least $N$ and at most $N$ : Whenever it is true that there are three bananas, it is also true that there are at least three bananas and at most three bananas. This entailment should not hold under the ignorance-as-entailment view, but should under the ignorance-as-implicature view, so these results provide empirical evidence for the hypothesis that the ignorance conveyed by superlatives is an implicature and not an entailment.

This also suggests that truth value judgment tasks may be a more robust tool for diagnosing entailment than validity judgment tasks, because they are less sensitive to pragmatic infelicity than validity judgment tasks. Recall that the experiment conducted by Geurts et al. (2010) tested parallel conditions using validity judgments, and obtained strikingly different results. There, the ignorance implication affected the judgments of around half of the participants, in contrast to the present experiment. The results from the two experiments are shown side by side in Figure 4. (The error bars on the right-hand side represent the $95 \%$ confidence interval of the mean.) The task of judging sentences true or false with respect to a depicted scenario appears to be a way of cutting through pragmatic effects, and getting to truth conditions. 
Diagnosing truth, interactive sincerity, and depictive sincerity

\begin{tabular}{lr}
\hline \multicolumn{1}{c}{ Conditions } & Percent "true" judgments (SD) \\
\hline There are at most $N X \mathrm{~s}$ in the picture. & $97.8 \%$ \\
There are fewer than $N+1 X \mathrm{~s}$ in the picture. & $97.8 \%$ \\
There are at least $N X \mathrm{~s}$ in the picture. & $100.0 \%$ \\
There are more than $N-1 X \mathrm{~s}$ in the picture. & $97.8 \%$ \\
There are at most $N-1 X \mathrm{~s}$ in the picture. & $3.3 \%$ \\
There are fewer than $N X \mathrm{~s}$ in the picture. & $1.0 \%$ \\
There are at least $N+1 X \mathrm{~s}$ in the picture. & $1.0 \%$ \\
There are more than $N+2 X \mathrm{~s}$ in the picture. & $3.3 \%$
\end{tabular}

Table 2 Percentage of participants that judged the sentences of Exp. 1 as true.

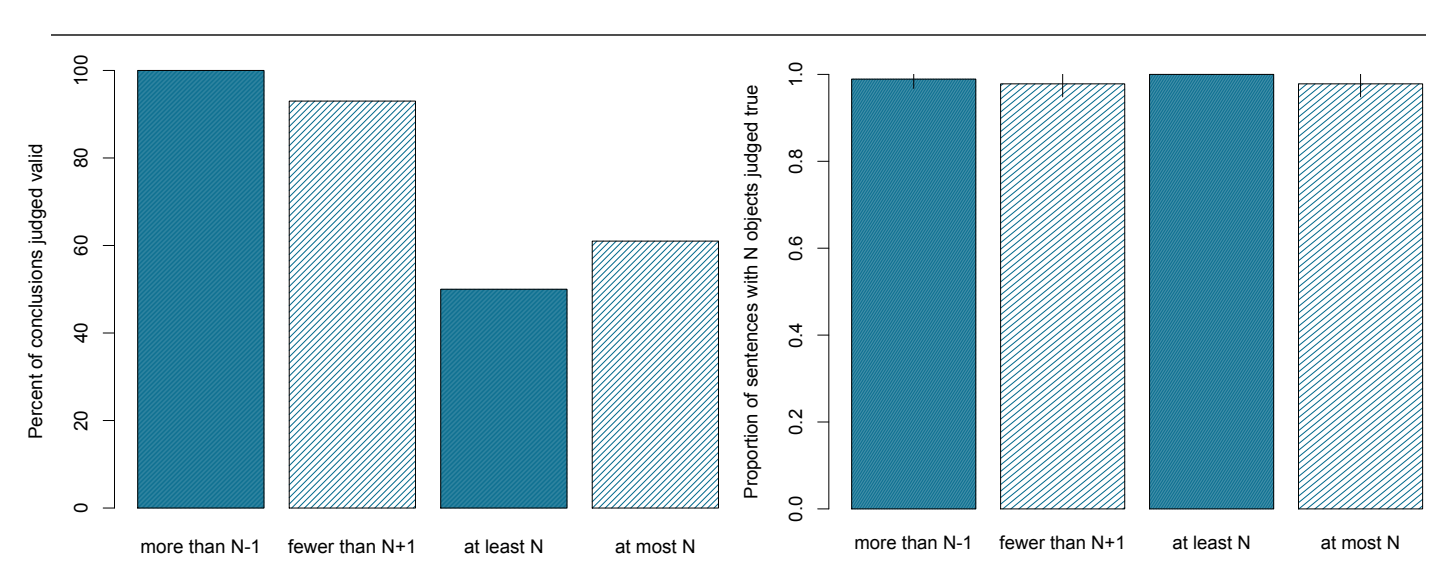

Figure 4 Results of Exp. 1 in Geurts et al. 2010 (left) and our Exp. 1 (right).

\subsection{Experiment 2}

\subsubsection{Methods}

The second experiment used the same design and methodology as Experiment 1, but the conditions were slightly different. In Experiment 1, the critical sentences depicted a range bounded by the quantity in the depicted scenario, whereas the critical sentences in Experiment 2 depicted a range bounded by one lower or higher than the depicted amount. For example, instead of testing for There are at least three bananas with a picture showing three bananas, we tested for There are at least two bananas. Table 3 shows the eight conditions used in this experiment.

As in the first experiment, each condition was judged four times per participant, and each participant saw 32 experimental stimuli and 32 fillers. Forty new participants were recruited using Amazon's Mechanical Turk. 


\begin{tabular}{ll}
\hline \multicolumn{1}{c}{ Critical } & \multicolumn{1}{c}{ Control } \\
\hline There are at most $N+1 X \mathrm{~s}$ in the picture. & There are at most $N-2 X \mathrm{~s}$ in the picture. \\
There are fewer than $N+2 X \mathrm{~s}$ in the picture. & There are fewer than $N-1 X \mathrm{~s}$ in the picture. \\
There are at least $N-1 X \mathrm{~s}$ in the picture. & There are at least $N+2 X \mathrm{~s}$ in the picture. \\
There are more than $N-2 X \mathrm{~s}$ in the picture. & There are more than $N+1 X \mathrm{~s}$ in the picture.
\end{tabular}

$N=$ amount of objects depicted, $X=$ type of object depicted.

Table 3 Conditions used in Experiment 2.

\begin{tabular}{lr}
\hline \multicolumn{1}{c}{ Conditions } & \\
\hline There are at most $N+1 X \mathrm{~s}$ in the picture. & Percent "true" judgments \\
There are fewer than $N+2 X \mathrm{~s}$ in the picture. & $76.1 \%$ \\
There are at least $N-1 X \mathrm{~s}$ in the picture. & $98.3 \%$ \\
There are more than $N-2 X \mathrm{~s}$ in the picture. & $98.3 \%$ \\
There are at most $N-2 X \mathrm{~s}$ in the picture. & $97.7 \%$ \\
There are fewer than $N-1 X \mathrm{~s}$ in the picture. & $4.4 \%$ \\
There are at least $N+2 X \mathrm{~s}$ in the picture. & $2.2 \%$ \\
There are more than $N+1 X \mathrm{~s}$ in the picture. & $2.8 \%$ \\
\end{tabular}

Table 4 Percentage of participants that judged the sentences of Exp. 2 as true.

\subsubsection{Results and discussion}

The full list of results is given in Table 4. As before, the sentences in the control conditions were judged to be false, meeting our pre-experimental expectations. Three out of the four modifiers behaved just as in Experiment 1, being consistently judged to be true under the new critical conditions. Yet the results obtained for at most $N+1$ were different. In spite of the fact that the sentences in this condition were expected to be compatible with the pictures shown, a substantial number of participants (24\%) judged them to be false. This is a statistically significant result. Using a mixed-effects binomial logit model with dummy-coded variables for each of the four critical conditions and random effects for subject and item, we found a significant contrast ( $p<0.0001$, based on the $t$-statistic) between the at most $N+1$ condition and the others, and no significant contrasts between the other conditions ( $p>0.4$ for each pairwise comparison).

This is a puzzling result, given what we have said so far. According to the truth conditions spelled out for at most in $\S 2$, a sentence with at most $N+1$ is true, though infelicitous, when there are $N$ objects. We would therefore expect participants to judge such a sentence as true in such a situation. As we will discuss in more detail below, we cannot explain the fact that these sentences elicit falsity judgments on 
Diagnosing truth, interactive sincerity, and depictive sincerity

\begin{tabular}{lr}
\hline \multicolumn{1}{c}{ Conditions } & Percent "true" judgments \\
\hline There are at most $N X \mathrm{~s}$ in the picture. & $97.8 \%$ \\
There are fewer than $N+1 X \mathrm{~s}$ in the picture. & $98.9 \%$ \\
There are at most $N+1 X \mathrm{~s}$ in the picture. & $44.3 \%$ \\
There are fewer than $N+2 X \mathrm{~s}$ in the picture. & $97.8 \%$
\end{tabular}

Table 5 Percentage of participants that judged the sentences of Exp. 3 as true.

the basis of their false ignorance implicature, because there are other sentences, involving at least, which also have a false ignorance implicature, which do not elicit judgments of falsity. In fact, as discussed in more detail below, neither existing approach to the ignorance implication predicts this rejection rate.

Before providing an explanation for this behavior, we wanted to see if this contrast concerning the findings for at most could be obtained within the same experiment. An explanation is given in $\$ 3.3 .2$.

\subsection{Experiment 3}

\subsubsection{Methods}

The third experiment zeroed in on the contrasting conditions from Experiments 1 and 2. We used fewer conditions, stimuli and participants, as the main goal was primarily to see if the contrast between Experiments 1 and 2 could be reproduced in one single experiment. Twenty participants were recruited through Mechanical Turk, and there were four conditions in this experiment, drawing from the contrasting conditions from Experiments 1 and 2: (i) at most $N$, (ii) at most $N+1$, (iii) fewer than $N+1$, and (iv) fewer than $N+2$. Each sentence type was judged four times per participant. There were 16 pictures, four of each quantity of objects from three to six, which were rotated through the conditions using a latin square design. To introduce variety and balance the number of sentences expected to be judged true and false, we included 32 fillers.

\subsubsection{Results and discussion}

As shown in Table 5 and Figure 5, the results are in line with the findings of the preceding experiments. Crucially, the judgments for the truth of sentences involving at most $N+1$ were significantly different from those for at most $N$ (44.3\% vs. $97.8 \%, p<0.0001$, using the same statistical techniques as above). This was the only significant contrast ( $p>0.7$ for all other pairwise contrasts). From this we can conclude that there is something special about this condition. 


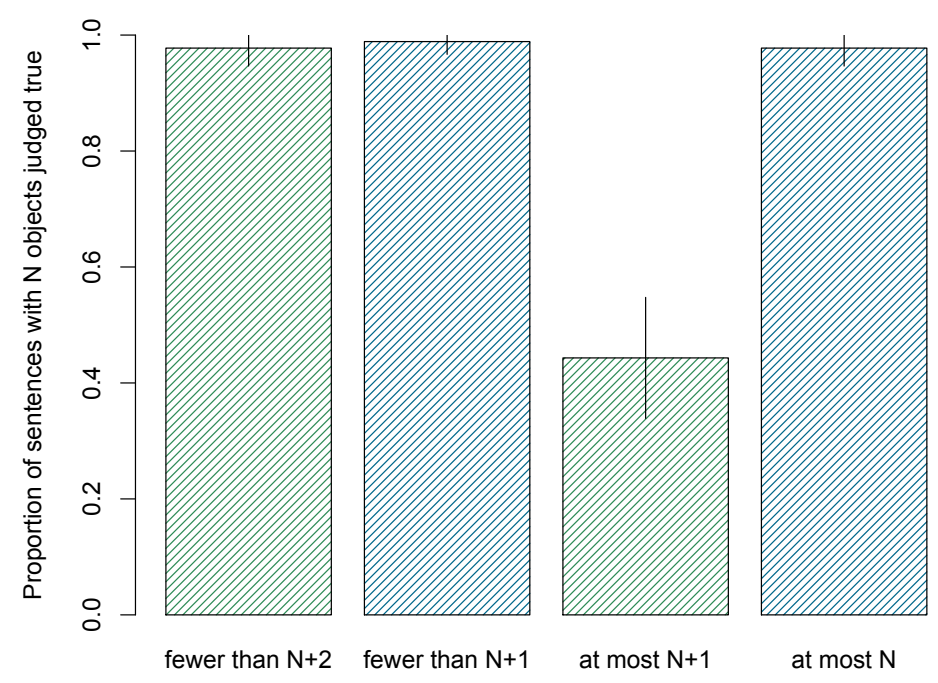

Figure 5 Results of Experiment 3 (graph).

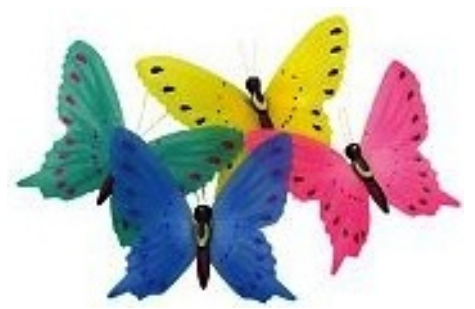

Figure 6 Example picture (four butterflies).

Let us consider whether one of the aforementioned approaches predicts the behavior we found across the first two experiments and replicated in the third. For the sake of discussion, let us focus on the following examples, in the context of the picture in Figure 6, where there are, as the reader can see, four butterflies:
a. There are at most four butterflies in the picture.
[97\% true]
b. There are at most five butterflies in the picture.
[44\% true]

According to Geurts \& Nouwen's (2007) theory, (14a) is true in case the speaker considers it possible that there are four butterflies and considers it impossible that there are more than four butterflies. Likewise for (14b), replacing 'four' with 'five'. If the speaker knows that there are exactly four butterflies (as in the picture), then (14b) should be false, because the speaker doesn't consider it possible that there are five butterflies. (14a) should be true, though, because the speaker certainly considers it possible that there are four butterflies if she knows that there are that 
many. So far, so good: The theory delivers a contrast where a contrast was found. The problem, however, is that the same reasoning should also apply to at least. In Experiment 1, speakers almost unanimously considered There are at least four butterflies in the picture to be true when there were four butterflies in the picture, but according to Geurts \& Nouwen's (2007) theory, this sentence should be false for exactly the same reason: The speaker believes it possible that there are more than four butterflies. Why don't speakers judge the at least four sentence to be false, by parity of reasoning, then?

Now we may ask if the inquisitive approach is better equipped to explain this contrast. According to Coppock \& Brochhagen (2013), superlative modifiers draw attention to a set of possibilities. In particular, There are at most five butterflies draws attention to the possibility that there are five butterflies, which is false in the situation where there are four butterflies. There are at most four butterflies does not draw attention to this false possibility. This might sound promising, but for reasons entirely analogous to those given in the previous paragraph, it does not suffice to explain what is going on. There are at least four butterflies also draws attention to the false possibility that there are five butterflies, and yet this sentence is unproblematically judged true. We thus arrive at the conclusion that neither approach accounts for this contrast based on the proposed properties of superlative modifiers.

So, why is There are at most five butterflies so likely to judged as false when there are four butterflies, even though at least four is straightforwardly true in such a situation? The answer, we propose, has to do with the one alternative explicitly mentioned on the surface. For example, There are at least four butterflies explicitly mentions the possibility that there are four butterflies, and There are at most five butterflies explicitly mentions the possibility that there are five butterflies.

In inquisitive semantics, there is a term for mentioning a possibility explicitly: highlighting. In general, a possibility in $\llbracket \varphi \rrbracket$ is highlighted if it is overtly expressed (Roelofsen \& van Gool 2010; Pruitt \& Roelofsen 2011; Farkas \& Roelofsen to appear). This notion has primarily been used to account for the contrast exhibited by polar questions, where it is assumed that the mentioned possibility is highlighted, and the response particles yes and no pick up the highlighted possibility. This explains the different inference patterns in (15) and (16).

(15) Is the door open?

a. Yes. $\Rightarrow$ the door is open

b. No. $\Rightarrow$ the door is closed

(16) Is the door closed?

a. Yes. $\Rightarrow$ the door is closed

b. No. $\Rightarrow$ the door is open 
These two questions convey the same inquisitive content: a choice between the possibility that the door is open, and the possibility that it is closed. Yet they differ with respect to what possibilities they highlight, thus influencing the inferences that arise from the different answers.

Now we are able to provide an answer to the question posed above: The difference between at most five and at least four is that the former highlights a false possibility, while the latter only brings attention to one. The at most five sentence highlights the possibility that there are five butterflies, which is plainly false in the context of Figure 6. The at least four sentence brings attention to the possibility that there are five butterflies, but does not highlight it; the at least four sentence highlights only the possibility that there are four (or more) butterflies, which is true.

To highlight a possibility known to be false, we suggest, goes against Gricean cooperativity. We propose to articulate the Gricean principle in question as follows.

\section{Maxim of Depictive Sincerity}

If $\varphi$ highlights a possibility $p$, then the speaker considers $p$ possible.

Depictive Sincerity seems to be a strong pragmatic requirement, compared to Interactive Sincerity. Recall that the results of Experiment 1, in contrast to Geurts et al.'s (2010) findings, show that violations of the Maxim of Interactive Sincerity do not influence truth-value judgments, although they do play a role for inference judgments. From this we concluded that truth-value judgments are less sensitive to pragmatics than other kind of tasks. Yet as the results for at most $N+1$ obtained in Experiments 2 and 3 show, even truth-value judgments are not safe from violations of the Maxim of Depictive Sincerity.

Comparatives like There are fewer than six butterflies do not violate the Maxim of Depictive Sincerity in the four-butterfly situation according to our theory. Recall that, following the treatment laid out in $\$ 2$, comparatives draw attention to a single possibility containing all alternatives under consideration. Since that possibility is the only one there is to begin with, that possibility is the only one that can be highlighted. (Following previous work on highlighting in inquisitive semantics, we assume that the highlighted possibility must be one of the elements of the denotation.) For example, There are fewer than six butterflies contains only the possibility of there being fewer than six butterflies, and this is the only one that can be highlighted. Thus, violating Depictive Sincerity is only possible for comparatives if the sentence is false. The highlighted possibilities for the comparative and superlative modifiers tested in Experiment 3 are depicted in Figure 7.

At this point, the reader may reasonably wonder whether a sentence like There are at least three butterflies violates the Maxim of Depictive Sincerity in the context of four butterflies, as it would seem to highlight the possibility that there are three butterflies. If a two-sided analysis ('exactly $N$ ') is given to numerals, this 
Diagnosing truth, interactive sincerity, and depictive sincerity

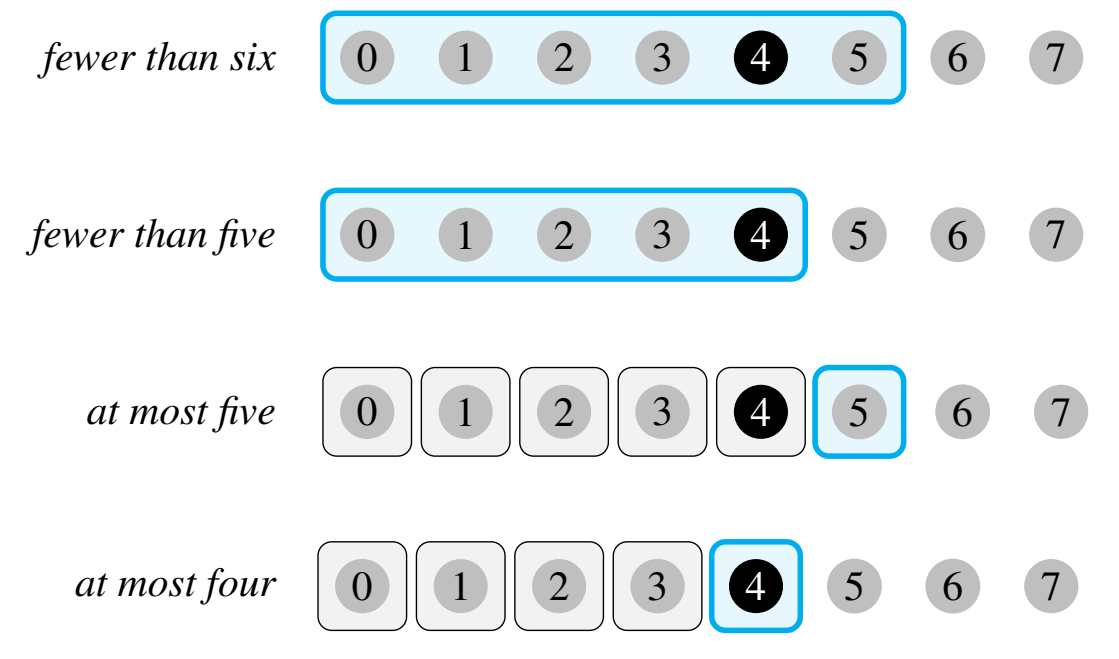

Figure 7 Highlighted (blue) and non-highlighted (grey) possibilities (on twosided reading, for aesthetic reasons), with $N=4$ objects in the picture. Only with at most five does the highlighted possibility fail to encompass the actual world (colored black).

sentence does indeed highlight a false possibility: $\left\{w_{3}\right\}$. But with a one-sided (' $N$ or more') analysis, the highlighted possibility is that there are three or more butterflies $\left(\left\{w_{3}, w_{4}, \ldots\right\}\right)$, and this is true (since it contains the actual world, $\left.w_{4}\right)$. We are thus driven to the auxiliary conclusion that numerals can receive one-sided interpretations, even if two-sided interpretations are also possible, as Kennedy (2012) argues.

\section{Conclusion}

We have provided experimental support for the hypothesis that the ignorance implication of superlatives is not an entailment, as on Geurts \& Nouwen's (2007) analysis, but rather conveyed via implicature, as advocated by Büring (2008), Cohen \& Krifka (2011), and Coppock \& Brochhagen (2013), among others. In Experiment 1, we found that at least $N$ and at most $N$ are judged to be true in case exactly $N$ is true very close to $100 \%$ of the time.

Further, a comparison of these experimental results with the ones obtained by Geurts et al. (2010) suggests that truth-value judgments are less sensitive to pragmatic infelicity than inference judgments. More concretely, it seems that ignorance implicatures do not affect truth-value judgments even though they do affect inference judgment tasks. Even though the critical sentences with superlative modifiers in 
Experiment 1 had false ignorance implicatures, they were still judged to be true. ${ }^{1}$

Still, the results from Experiments 2 and 3 show that even truth-value judgment tasks are not completely impervious to pragmatics. In a situation with $N X \mathrm{~s}$, it was judged false that there are 'at most $N+1 X \mathrm{~s}$ ' at substantial rates (24\% in Experiment 2 , and $56 \%$ in Experiment 3 ). We argued that this cannot be due to the ignorance implicature, and that it has to do with the fact that the alternative that is explicitly mentioned is false. We posited a Gricean maxim (the Maxim of Depictive Sincerity) which rules against mentioning a possibility known to be false, and concluded that violations of this maxim - unlike violations of the Maxim of Interactive Sincerity - can cause true sentences to be judged as false. This opens up the general question of which Gricean maxims are so strong that they affect truth value judgments, and which are not, and why.

\section{References}

Biezma, María. 2013. Only one at least: Refining the role of discourse in building alternatives. In University of Pennsylvania Working Papers in Linguistics, vol. 19 1, 11-19. Penn Linguistics Club.

Büring, Daniel. 2008. The least at least can do. In Charles B. Chang \& Hannah J. Haynie (eds.), 26th West Coast Conference on Formal Linguistics, 114-120. Somerville, MA: Cascadilla Press.

Ciardelli, Ivano, Jeroen Groenendijk \& Floris Roelofsen. 2009. Attention! Might in inquisitive semantics. In Satoshi Ito \& Ed Cormany (eds.), Semantics And Linguistic Theory (SALT) XIX, 91-108. CLC Publications.

Ciardelli, Ivano, Jeroen Groenendijk \& Floris Roelofsen. 2012. Inquisitive semantics. NASSLLI 2012 lecture notes.

Cohen, Ariel \& Manfred Krifka. 2011. Superlative quantifiers as modifiers of metaspeech acts. In Barbara H. Partee, Michael Glanzberg \& Jurgis Skilters (eds.), The Baltic International Yearbook of Cognition, Logic and Communication, vol. 6, 1-56. New Prairie Press.

Coppock, Elizabeth \& Thomas Brochhagen. 2013. Raising and resolving issues with scalar modifiers. Semantics \& Pragmatics 6(3). 1-57.

1 One might wonder whether the ignorance implicature even arises in the truth-value judgment context. We have no reason to believe that it does not, and we have been assuming that participants judge the sentence to be true despite pragmatic infelicity. But the data is also consistent with the possibility that this implicature does not arise. Indeed, in the truth-value judgment task, it is not entirely clear who the speaker is. Is it the computer? The experimenter? The participant in the experiment? Is there even a speaker, given that the sentence is being mentioned, rather than used? This unclarity could mean that there is no ignorance implicature. Thanks to Matthijs Westera and Laura Staum Casasanto for discussion of this issue. 
Diagnosing truth, interactive sincerity, and depictive sincerity

Cummins, Chris \& Napoleon Katsos. 2010. Comparative and superlative quantifiers: Pragmatic effects of comparison type. Journal of Semantics 27. 271-305.

Farkas, Donka \& Floris Roelofsen. to appear. Polar initiatives and polarity particle responses in an inquisitive discourse model. Language .

Geurts, Bart, Napoleon Katsos, Chris Cummins, Jonas Moons \& Leo Noordman. 2010. Scalar quantifiers: Logic, acquisition, and processing. Language and Cognitive Processes 25.

Geurts, Bart \& Rick Nouwen. 2007. At least et al.: The semantics of scalar modifiers. Language 83. 533-559.

Groenendijk, Jeroen \& Floris Roelofsen. 2009. Inquisitive semantics and pragmatics. Presented at the Workshop on Language, Communication, and Rational Agency at Stanford, May 2009, available via www.illc.uva.nl/inquisitivesemantics.

Kaplan, David. 1999. The meaning of ouch and oops. Lecture presented at the University of California at Berkeley.

Kennedy, Chris. 2012. A scalar semantics for scalar readings of number words. Semantics Archive.

Nouwen, Rick. 2010. Two kinds of modified numerals. Semantics \& Pragmatics 3. $1-41$.

Pruitt, Kathryn \& Floris Roelofsen. 2011. Disjunctive questions: prosody, syntax, and semantics. Presented at a seminar at the Georg August Universität Göttingen, April 2011.

Roelofsen, Floris \& Sam van Gool. 2010. Disjunctive questions, intonation, and highlighting. In Maria Aloni, Harald Bastiaanse, Tikitu de Jager \& Katrin Schulz (eds.), Logic, Language and Meaning: Selected Papers from the 17th Amsterdam Colloquium, 384-394. Berlin: Springer.

Elizabeth Coppock

Department of Philosophy, Linguistics and Theory of Science

University of Gothenburg

Box 200

40530 Gothenburg, Sweden

eecoppock@gmail.com

Thomas Brochhagen

Abteilung für Allgemeine Sprachwissenschaft

Heinrich-Heine-Universität Düsseldorf

Universitätsstrasse 1

40225 Düsseldorf, Germany

thomas.brochhagen@hhu.de 